\title{
Civilisations
}

Revue internationale d'anthropologie et de sciences

humaines

$51 \mid 2004$

Religions transnationales

En guise de réponse : les Assemblées de Dieu du Burkina Faso et la trans-nationalité du pentecôtisme

\section{Pierre-Joseph Laurent}

\section{Q OpenEdition}

\section{Journals}

\section{Édition électronique}

URL : http://journals.openedition.org/civilisations/701

DOI : 10.4000/civilisations.701

ISSN : 2032-0442

\section{Éditeur}

Institut de sociologie de l'Université Libre de Bruxelles

\section{Édition imprimée}

Date de publication : 1 janvier 2004

Pagination : 201-223

ISSN : 0009-8140

\section{Référence électronique}

Pierre-Joseph Laurent, « En guise de réponse : les Assemblées de Dieu du Burkina Faso et la transnationalité du pentecôtisme », Civilisations [En ligne], 51 | 2004, mis en ligne le 07 janvier 2009, consulté le 30 avril 2019. URL : http://journals.openedition.org/civilisations/701 ; DOI : 10.4000/ civilisations.701

Ce document a été généré automatiquement le 30 avril 2019.

(c) Tous droits réservés 


\title{
En guise de réponse: les Assemblées de Dieu du Burkina Faso et la trans- nationalité du pentecôtisme
}

\author{
Pierre-Joseph Laurent
}

1 Qui n'a pas été surpris par le développement sans précédent du religieux dans les villes africaines? Depuis deux décennies, une nouvelle vague pentecôtiste, essentiellement urbaine, connaît une expansion importante, estimée de par le monde à près de cent millions de fidèles (Corten et Marshall-Fratani, 2001). Ce qui frappe à la lecture des travaux consacrés aux pentecôtismes au Bénin, au Brésil, au Mexique, à Haïti, en Afrique du Sud, au Ghana, au Burkina Faso, ou encore au Nigeria (Bastien, 2001; Corten, 1995 ; Corten et Mary, 2000 ; de Surgy, 2001 ; Laurent et Mary, 2001 ; Laurent, 2003a ; MarshallFratani, 2001; Tonda, 2002; Willaime, 1999), par delà l'extrême variabilité, c'est la relative similitude, d'une part, dans l'expression de la souffrance par la symbolique du corps possédé et soulagé grâce à l'exorcisme que pratiquent les religieux, et, d'autre part, dans l'appropriation de la Bible, non à travers une lecture savante, mais par l'expérience personnelle de la puissance divine et l'émotion de la communauté croyante. Dans ce sens, il est possible d'interpréter «l'Esprit de pentecôte» comme «la ruse d'un Esprit populaire », où, par une appropriation émotionnelle chez un fidèle inspiré de l'Esprit Saint, l'ordre établi et plus particulièrement celui des pasteurs est sans cesse remis en question. Serions-nous en présence d'une expression déjà mondialisée de la souffrance, du malheur et de la quête d'un bonheur hic et nunc dans une frange de laissés-pourcompte de la globalisation?

2 L'idée centrale de l'ouvrage est que le pentecôtisme et en l'occurrence l'Eglise des Assemblées de Dieu du Burkina Faso (AD/BF), qui implique chez les convertis le sentiment d'appartenir à une communauté transnationale, produit des ressources inédites pour trouver de nouveaux chemins dans un moment particulier de réforme de la culture, celle qui est liée à la modernité globalisée. L'objectif de mes recherches a consisté à déterminer la nature de ces ressources et la teneur du bricolage des identités ainsi produites. L'enjeu 
descriptif et théorique qui consiste à lier le local au global reste difficile à mettre en œuvre ${ }^{1}$. C'est pourquoi il est incontournable, pour se poser la question du niveau global, de partir d'observations de longue durée sur le terrain. Pour cela, il importe de décrire localement, avec minutie, les transformations des systèmes de pensée. A mon niveau, le préalable fut donc, au risque d'être réduit aux conjectures, la maîtrise de la société coutumière mossi, ainsi que de ses manières de se représenter le monde.

3 Le contexte de l'analyse est donc bien celui de la transformation de la société paysanne mossi du Burkina Faso, désormais perçue par les pentecôtistes comme une "vieille peau » dont ils veulent s'éloigner. Il s'agit de rendre compte des effets de l'urbanisation et donc du passage du village à la ville. Il semble aujourd'hui établi que d'ici 2025, $60 \%$ de la population de l'Afrique de l'Ouest sera urbaine. Au-delà de cette prévision, ce qui se transforme avec l'urbanisation récente et rapide de cette région d'Afrique et l'émergence des villes, c'est la manière de vivre en commun. Lorsqu'on change de lieu pour passer du village à la ville, on change aussi de logique, transitant d'une situation où l'on se connaît personnellement, à celle d'un anonymat qui conduit à une certaine atomisation du social. Ce sont les anciens modes de régulation de la vie collective qui subissent des transformations, des bouleversements, des adaptations, pour répondre au défi de la recherche de la meilleure sécurité sociale et économique pour les groupes de populations qui débarquent dans les zones en voie d'urbanisation. Les populations, confrontées à l'urgence de réguler la vie en commun (accès au foncier, sécurité des biens et des personnes, approvisionnement, eau potable, gestion des déchets...), produisent (sans forcément attendre les injonctions de l'Etat) des modes divers d'organisation collective, dans lesquels les communautés protestantes jouent un rôle. Ainsi, dans les quartiers populaires de Ouagadougou, dans les petites villes, mais aussi désormais dans les villages du plateau mossi, les Eglises protestantes s'affirment aujourd'hui comme un lieu de grande socialité et se montrent capables, à travers les manifestations miraculeuses de l'Esprit Saint relayées par des croyants-guérisseurs ${ }^{2}$, de résoudre solitude, maladies, souffrance, chômage et adversité.

Désormais, dans les villes émergentes du Burkina Faso, on danse, le samedi soir, dans les bars sur les mêmes rythmes que dans les autres villes du monde. Autrement dit, les nouveaux centres urbains deviennent des lieux de transformation rapide des identités coutumières et le creuset de nouvelles pratiques économiques au sein desquelles les mouvements religieux se taillent une place notoire. C'est dans ce sens que la doctrine et la pratique pentecôtiste offrent une ouverture sur le monde que la modernité promet. Cette ouverture porte sur une transformation radicale du «moi» et une nouvelle identité collective laquelle parvient à satisfaire les fidèles en dépit d'un contexte où la participation de beaucoup à la consommation et l'accès aux richesses se réduit souvent à une "vitrine». Par la conversion au pentecôtisme, la transformation du monde à un niveau individuel produit l'imaginaire nécessaire à une inspiration qui devrait conduire le fidèle à croire qu'une autre vie est possible, là où la forme collective du changement s'exprime dans la " guerre contre Satan ».

5 Les convertis modifient leurs perceptions des relations aux autres en adoptant une vision essentiellement dualiste du monde. La société coutumière, "officiellement» défaite, équivaut désormais à un monde démoniaque, dont il convient d'avoir l'audace de se distancier, au même titre d'ailleurs que de «la société (moderne)» identifiée «au monde ", c'est-à-dire à un univers corrompu, où règnent la violence, la peur et la défiance (Laurent, 1998a). En référence à la Bible, les protestants décodent dorénavant les rapports 
sociaux en termes de bien et de mal ${ }^{3}$. De sorte que les convertis se meuvent dans un entre-deux-mondes, dotés qu'ils sont de cette capacité de discernement. Cette conversion que je qualifierai de "bien tempérée ", à la laquelle renvoie au mieux le concept de bricolage ${ }^{4}$, met l'accent sur une prise de distance relative vis-à-vis de l'entraide lignagère, tout autant que des formes d'insécurité qu'a pu engendrer l'Etat post-colonial. Elle conduit à l'invention d'un mode d'existence un peu plus individuel, tout en tentant de pallier aux manquements de l'Etat-nation.

6 Le pentecôtisme est la principale transnationalisation religieuse du vingtième siècle. Et dans ce sens, ce travail monographique montre comment les ressources inédites qu'offre le pentecôtisme sont appropriées par des groupes de population mossi du Burkina Faso, même les plus isolés en brousse, en modifiant les catégories d'espace et de temps. Les communautés protestantes se composent de croyants, lesquels, doués d'une capacité d'action sur la scène locale, vont "comprendre " d'abord et ré-articuler ensuite des éléments transnationaux et exogènes du pentecôtisme, comme autant de ressources qu'ils utiliseront dans leurs propres stratégies de survie, confrontés à un environnement en proie à de profondes transformations.

7 Plus de dix années d'enquêtes ont été nécessaires pour que je parvienne à décrire avec une égale précision la complexité des pratiques sociales et des représentations culturelles des paysans mossi «protestants » et l'univers du pentecôtisme des villes émergentes du Burkina Faso. Le recours à une monographie, classique dans sa conception, - elle repose sur la description des formes de l'alliance, des conceptions du pouvoir, de la maladie et de la guérison - me permet d'établir, étape par étape, la manière dont les apports du pentecôtisme vont conduire les fidèles mossi à mettre à bonne distance le communautarisme traditionnel, de même que «le monde» (soit une forme de la modernité qu'ils désignent comme corrompue, règne de la violence, de la peur et de la défiance), pour parvenir à élaborer de nouvelles synthèses identitaires. Comme le souligne Frédéric Moens, l'étude du pentecôtisme constituait une excellente opportunité de poursuivre mes travaux sur les processus de transformation de la société mossi et sur son entrée dans la modernité globalisée. Au-delà, il s'agit d'apporter une contribution à la compréhension de la "pentecôtisation » de groupes de population. C'est donc à dessein que l'historique de l'Eglise des Assemblées de Dieu (AD) est d'emblée synthétisée dans un chapitre d'ouverture car l'histoire missionnaire de l'implantation du pentecôtisme dans cette région de l'Afrique, les intrigues filandreuses liées à l'institutionnalisation des multiples dénominations pentecôtistes, ainsi que leurs processus de segmentation, pour importants et intéressants qu'ils soient, ne constituent toutefois pas l'objet primordial de cet ouvrage ${ }^{5}$.

\section{Processus d'expansion des Assemblées de Dieu du Burkina Faso}

8 La mouvance pentecôtiste du Burkina Faso est estimée à plus de 700.000 personnes ${ }^{6}$, soit $5,8 \%$ de la population totale évaluée à 12 millions d'habitants (2003). Pour leur part, les AD/BF compteraient (2002) 2.600 églises $^{7}$, plus de 2.500 pasteurs $^{8}, 600.000$ membres (soit $5 \%$ de la population totale) $)^{9}, 6$ écoles de formation biblique et un Institut supérieur de théologie ${ }^{10}$, ce qui représenterait approximativement $86 \%$ de la mouvance pentecôtiste de ce pays qui se morcelle, pour les $14 \%$ restant, en plus de 20 appellations différentes, pour les plus connues et uniquement pour la capitale. Ces dernières, à la différence des 
$\mathrm{AD} / \mathrm{BF}$, se concentrent plutôt dans les principales villes du pays et sont pour ainsi dire quasi absentes des zones rurales du plateau mossi. Par ailleurs, les dénominations pentecôtistes sont en augmentation suite notamment à la décision (avril 2003) des autorités d'octroyer plus aisément des reconnaissances officielles aux nouveaux mouvements religieux qui en font la demande. J'ai montré qu'en plus d'être la plus ancienne Eglise pentecôtiste de ce pays, les $\mathrm{AD}$ ont engendré, à la suite de dissidences, quelques autres dénominations pentecôtistes: la Mission Apostolique, l'Eglise Apostolique, l'Eglise de Pentecôte (issue d'une sous-division ${ }^{11}$ ), ou encore récemment l'Eglise du Réveil Spirituel et l'Eglise Réformée des AD (Laurent, 1999). Au Burkina Faso, parler des "Américains", pour reprendre le vocable utilisé par la population, désigne indistinctement les fidèles des AD et les «protestants ». Les AD constituent le socle du pentecôtisme dans ce pays. Cependant, c'est donc plutôt par leur efficacité et leur capacité d'organisation que les $\mathrm{AD} / \mathrm{BF}$, minorité agissante, jouent un rôle notoire au sein de la société burkinabé, voire auprès de certaines Eglises sœurs d'Afrique de l'Ouest.

L'installation des AD/BF remonte à 1921. Depuis le début du siècle et à partir de l'Eglise mère, aujourd'hui située à Springfield dans le Missouri aux Etats-Unis, s'organisent plusieurs implantations missionnaires sur le continent africain. Selon la littérature officielle des $\mathrm{AD}$, les membres et les adhérents atteindraient 48 millions de personnes de par le monde. L'Eglise compterait 250.000 lieux de cultes répartis dans 198 pays, 290.000 pasteurs et près 2.000 écoles bibliques ${ }^{12}$. Ces données sont toutefois à considérer avec précaution, d'abord parce qu'elles émanent des Eglises elles-mêmes et ensuite en raison de la différence entre les sources consultées: ainsi les statistiques mondiales publiées dans le journal officiel des $\mathrm{AD} / \mathrm{BF}$ donnaient le chiffre de 32 millions de membres (2000) ${ }^{13}$. Les $\mathrm{AD}$ seraient ainsi le plus important mouvement pentecôtiste de par le monde. Les communautés les plus importantes, en dehors du Burkina Faso, se situent au Brésil avec près de 17 millions de fidèles ${ }^{14}$, aux Etats-Unis avec 2,6 millions d'adhérents ${ }^{15}$, les Eglises du Nigeria et de Corée comptent plus d'un million et demi de convertis (Lucas, 1999) ${ }^{16}$, celles du Mexique, d'Argentine (Corten, $1995: 15)^{17}$ rassemblaient déjà en 1995 chacune environ un demi-million de fidèles. Les AD appartiennent au Mouvement Pentecôtiste Moderne qui émerge aux Etats-Unis dès le début du $\mathrm{XX}^{\mathrm{e}}$ siècle, au sein des communautés de Réveil du Saint-Esprit (Laurent, 2003a: 29-33). Pour l'Afrique, les données fournies par l'Alliance des AD d'Afrique (AADA), présidée depuis août 2000 par le pasteur Pawentaoré, responsable de l'Eglise du Burkina Faso ${ }^{18}$, signalent une implantation dans 39 pays, 24.016 lieux de culte, 23.282 pasteurs, 305 missionnaires, pour environ 10 millions de membres aujourd'hui ${ }^{19}$. En Afrique les Eglises les plus importantes sont : le Nigeria, 1,7 million (soit $1,5 \%$ de la population totale), 9 écoles bibliques ; le Burkina Faso (5 \%) (cf. ci-dessus); le Kenya, 560.000 (2,9\%), 2 écoles ; la Côte d'Ivoire 500.000 (3,1\%), 2 écoles ; la Tanzanie, 400.000, 4 écoles (1,4\%); le Mozambique, 295.000 (1,5\%), 1 école; la Zambie, 154.800 (1,6\%), 2 écoles ; le Bénin, 117.500 (1,8\%), 2 écoles ; le Togo, 113.000 (2,2\%), 2 écoles ; le Ghana, 95.000 (0,6\%), 3 écoles ; l'Afrique du Sud, 74.058 (0,02\%), 3 écoles $^{20}$.

Les chiffres étant rappelés, je peux à présent aborder l'objet essentiel de ce point. Dans un paysage pentecôtiste fréquemment traversé par des mouvements de segmentation et l'apparition de nouvelles Eglises, la domination des AD sur la mouvance pentecôtiste du Burkina Faso demande des éclaircissements historiques qu'il faut aller chercher dans l'histoire longue de la société mossi et la pauvreté objective du plateau mossi ${ }^{21}$. Avec ces $5 \%$ de la population nationale, situation unique en Afrique pour les $A D$, l'Eglise à l'opportunité de jouer un rôle dans le paysage politique du pays ${ }^{22}$, ainsi qu'une certaine 
ascendance sur les Eglises soeurs d'Afrique. A ce titre, seules les Eglises du Kenya (3\%) et de Côte d'Ivoire (avant sa partition) pourraient prétendre à un tel rôle. Au Burkina Faso, les $\mathrm{AD}$ sont aujourd'hui considérées comme une Eglise nationale, instituée, à côté de l'Eglise catholique et des grandes fédérations musulmanes.

11 Sandra Fancello rappelle avec justesse que la zone d'expansion des Eglises pentecôtistes en Afrique de l'Ouest est aujourd'hui essentiellement concentrée sur un axe fort qui va de Lagos au Ghana. Toutefois, du point de vue historique, les informations qu'elle fournit selon lesquelles l'Eglise des $\mathrm{AD}$ est la première Eglise pentecôtiste entrée au Ghana (en 1931) à la faveur du travail de missionnaires installés au Burkina Faso sont utiles et suffisent déjà à souligner que les $\mathrm{AD}$ ont fait du Burkina Faso une "plaque tournante " pour la région.

12 Ainsi, sans entrer dans les détails ${ }^{23}$, comment ne pas signaler et en même temps s'étonner de la présence de pasteurs mossi dès 1936 au Togo ${ }^{24}$, en 1945 au Bénin ${ }^{25}$, en 1945 au Sénégal ${ }^{26}$, en 1935 au Niger $^{27}$, en 1931 au Ghana ${ }^{28}$, en 1958 en Côte d'Ivoire ${ }^{29}$ ? Par ailleurs, comment expliquer que pendant plusieurs années le pasteur Daniel Pasgo fut le premier président des $\mathrm{AD}$ du Daho-Togo ${ }^{30}$, que le pasteur Jean Bande est l'actuel vice-président de la Faculté de théologie des AD à Lomé et en fut le doyen de 1988 à 1998 (ancien ESTAO) ${ }^{31}$, que le pasteur Emmanuel Kiemtore fut animateur à la radio « Jésus vous aime » à Lomé au Togo $^{32}$, qu'un pasteur mossi vient de prendre la direction des $A D$ du Sénégal ${ }^{33}$, que le pasteur Jean-Ernest Sawadogo assume, depuis le début, la direction des $\mathrm{AD}$ au Niger ${ }^{34}$, que le pasteur Tiiga fut le premier président des AD du Ghana ${ }^{35}$, que le pasteur Abel Konseiga a été nommé président de la région de Guinée ${ }^{36}$ et que le pasteur Adama Ouedraogo fut pendant longtemps le président des $\mathrm{AD}$ de Côte d'Ivoire ${ }^{37}$ ? La présence de pasteurs burkinabé à ces postes de responsabilité ne méritait-elle pas que j'y consacre quelques lignes? Le pasteur Daniel Delma, secrétaire général adjoint des AD du Burkina Faso et coordinateur de la troisième révision de la traduction de la Bible m'affirmait encore en avril 2003 que la dissémination des $\mathrm{AD}$ dans les pays de l'Afrique de l'Ouest francophone, essentiellement, s'est historiquement réalisée à partir du Burkina Faso. Pour appuyer cette affirmation, j'évoquerais seulement ici un détail glané lors des entretiens que j'ai eu avec le pasteur Daniel Compaoré (sorti de la seconde promotion de l'école biblique de Koubri en 1947, président des AD de 1967 à 1979 et responsable jusqu'en 1998 de l'imprimerie des AD). Cette imprimerie, qu'il dirige dès 1949, servira progressivement, m'expliqua-t-il, à toutes les AD installées en Afrique de l'Ouest, au Bénin, au Togo, en Côte d'Ivoire, au Sénégal, au Ghana et au Burkina Faso. Ouagadougou devint alors le centre de production de la littérature des AD pour l'Afrique de l'Ouest. En 1973, une partie de l'atelier est transféré au Ghana. En 1986, de nouvelles machines viennent renforcer l'atelier de Ouagadougou (financement mission américaine) ${ }^{38}$.

13 Si les précisions fournies par Sandra Fancello corroborent mes observations selon lesquelles l'expansion des $\mathrm{AD}$, soutenue par la contribution des pasteurs mossi, s'est faite d'abord "en se calquant sur les itinéraires de migrations mossi vers les pays côtiers " (surtout la Côte d'Ivoire, où, par exemple, plus d'une centaine de pasteurs mossi ont exercé avant les événements de ces dernières années ${ }^{39}$, et le Ghana), son approche comparative des modes d'expansion fonctionne comme une heuristique : l'auteur y décrit un rapport de similitude inversée entre la Church of Pentecost du Ghana et les AD/BF.

En revanche l'argumentation principale de Joël Noret consacrée à ma "surestimation » du rôle des pasteurs et du pentecôtisme mossi en Afrique de l'Ouest est singulière. Il est bizarre de me reconnaître à la fois la pertinence du rôle pionnier des débuts tenu par les 
pasteurs mossi, isolés ou en appui à des missionnaires américains ou français dans la propagation des $\mathrm{AD}$ dans les pays limitrophes du Burkina Faso (voir dans le texte de Joël Noret le troisième point de son argumentation) et en même temps prétendre que je surévalue ce rôle, et ce, totalement en dehors de mes propos. En effet, dans un ouvrage de plus quatre cents pages, j'en consacre quarante à l'histoire de l'implantation des $\mathrm{AD}$ au Burkina Faso. De plus, ce chapitre ne fait qu'évoquer - car ce n'est pas l'objet de mon livre - , le rôle, pourtant important, des AD/BF dans l'expansion de l'Oeuvre en Afrique; j'y consacre trente lignes ( $c f$. pages $29,51,55-56$ et 65 , soit deux-tiers de page) : est-ce finalement trop ou trop peu?

En résumé, il est évident que du point de vue historique, l'Eglise des $\mathrm{AD} / \mathrm{BF}$ a joué un rôle notoire dans l'expansion du mouvement en Afrique de l'Ouest et plus largement, mais aussi plus modestement, dans celui du pentecôtisme. Même si elle n'est pas la seule, il est incontestable que les $\mathrm{AD}$ ont fait du Burkina Faso une « plaque tournante » pour la région.

Pour rendre compte de la place particulière des $\mathrm{AD} / \mathrm{BF}$ à l'intérieur du Burkina Faso et de sa participation à l'expansion de l'Oeuvre en Afrique de l'Ouest, j'avance, dans le livre, l'idée que l'organisation autoritaire, hiérarchisée et centralisée de la société mossi a joué un rôle sur la construction institutionnelle de l'Eglise. Il est un fait qu'avec $5 \%$ de la population nationale et près de $90 \%$ de la mouvance pentecôtiste du pays, les $\mathrm{AD} / \mathrm{BF}$ ont pu s'imposer comme une Eglise instituée, capable de tenir un rôle sur la scène sociale et politique du pays. Ce mode de fonctionnement s'oppose à celui des petites communautés pentecôtistes liées aux charismes de leurs leaders. Chez les AD/BF, les fidèles et les pasteurs sont tenus d'obéir aux règles instituées par les responsables de l'Eglise. L'ancienneté de l'implantation de l'Eglise en pays mossi, l'autoritarisme des dirigeants et le contrôle de la cohérence doctrinaire ont très tôt joué en faveur de la structuration et de l'institutionnalisation du mouvement.

Par ailleurs, la pauvreté objective du plateau mossi, occupé par une population paysanne inquiète de sa survie et à la recherche de ressources (encline à la migration), est un facteur explicatif de l'implication des fidèles dans l'Oeuvre; ils y trouvent, peut-être plus qu'ailleurs, les possibilités d'une existence acceptable. Les mouvements migratoires de la population mossi, vers les pays " côtiers » surtout, constituent un autre facteur ayant favorisé l'expansion des $\mathrm{AD}$ dans les pays limitrophes à partir du Burkina Faso. Le pentecôtisme des AD s'est trouvé dans un rapport dynamique, qu'il a su exploiter, avec la société mossi qui fut expansionniste ( $c f$. le mode de fonctionnement des commandements mossi), et dont les chefs savaient se montrer autoritaires et impitoyables vis-à-vis d'une population soumise qui a toujours utilisé la migration comme mode de subsistance. La force des $\mathrm{AD} / \mathrm{BF}$ est d'incarner en milieu rural "le groupe de la sortie du groupe » (Laurent, 1998b) et donc une forme de la modernité, et en même temps, dans les villes émergentes, l'Eglise donne aux convertis le sentiment d'appartenir à une communauté transnationale qui leur offrent une ouverture sur le monde que la modernité globalisée promet dans un contexte parfois d'extrême pauvreté. 


\section{Le contexte : spécificités du pentecôtisme au Burkina Faso}

\section{Méfiance à l'égard de la doctrine de la prospérité}

La « théologie de la prospérité » accompagne la naissance des Eglises néo-pentecôtistes et une évolution socio-économique majeure : les « vrais » chrétiens qui se désintéressaient «du monde », semblent désormais se satisfaire "d'une accommodation au monde ». La «théologie de la prospérité » équivaut à défier Dieu, c'est-à-dire à l'aimer aveuglément, au point de lui offrir au delà de ce qu'on possède et lui prouver ainsi sa confiance. Ces sacrifices financiers permettent d'accéder à ce à quoi le fidèle estime avoir droit (le bonheur hic et nunc), soit les bénédictions que Jésus a rendues accessibles par son sacrifice. Le fidèle donne avec fierté et accède au sentiment d'agir selon sa propre volonté (Fialho-Costa, 2002).

Avec la "théologie de la prospérité", nous sommes en présence d'une religiosité populaire où s'affirme l'idée d'endetter la divinité à travers des dons, et plus précisément une forme de dons rusés (silim kouni en moore: Laurent, 1998b), avec l'espoir de recevoir plus un jour. Et dans ce sens, « la théologie de la prospérité » s'apparente à la formule do ut des, du donner pour recevoir. Ceci évoque les formes de l'entraide, la logique de l'endettement et la recherche de la dépendance de l'entourage, en guise d'investissement sur un long terme. Donner équivaut ici à s'acquitter de la dîme (wênnam puîire : litt. la part de Dieu), afin de recevoir la bénédiction divine. En d'autres mots, plus on donne et plus on gagne en bénédiction. A contrario, ne pas donner à Dieu égalerait à donner au diable.

Le processus socioculturel sous-jacent à la «théologie de la prospérité » semble aller de soi pour les franges les plus populaires des adeptes qui, en quelque sorte, entrainent les $\mathrm{AD} / \mathrm{BF}$ dans une "pentecôtisation" (ce qui équivaut à un pentecôtisme de deuxième génération), tandis que l'engagement vertueux des «soldats de Dieu » à la construction de l'Oeuvre résulte plutôt d'une minorité instruite. Les $\mathrm{AD} / \mathrm{BF}$, implantées dans le pays depuis près de quatre-vingts ans, révèlent ici une de leurs caractéristiques majeures. Cette singularité tient dans leur caractère institué, où les $\mathrm{AD} / \mathrm{BF}$ se montrent soucieuses de leur respectabilité et de leur place dans la société burkinabé. Les pasteurs, en ville comme au village, chacun pour leur compte, s'affichent comme des notables. Dès lors, c'est principalement en ville, plutôt qu'à la campagne, à l'instigation de fidèles ou de jeunes pasteurs non encore établis, et non pas des responsables de l'Eglise, que les cultes se sont enflammés par les manifestations de plus en plus nombreuses du Saint-Esprit. Il en est de même de l'apparition des cultes de délivrance animés par des croyantsguérisseurs exerçant parfois leurs dons à la lisière de l'Eglise instituée. En bref, la «pentecôtisation » des $\mathrm{AD} / \mathrm{BF}$, avérée depuis la dernière décennie, résulte plus des fidèles que de l'élite, laquelle perçoit ce processus à la fois comme une contestation interne, une perte de contrôle sur les destinées de l'Eglise et un risque de segmentation.

21 Faisant plutôt référence aux « entrepreneurs religieux » qui tirent profit de la crédulité populaire, et dont il se méfie, le président des $\mathrm{AD}$ estime que la «théologie de la prospérité » ne peut pas fonctionner au Burkina Faso car cette croyance stigmatise la pauvreté. Le pasteur Philippe Ouedraogo, responsable de la région de Ouagadougou et membre du bureau exécutif de l'Eglise, estime que la «théologie de la prospérité » 
constitue une dérive dans la mesure où elle ne figure pas dans la Bible. Le refus de cette théologie s'appuie sur la récusation de la liaison de la pauvreté au péché.

Pasteur Philippe (membre du bureau exécutif):Ceux qui prônent aujourd'hui cette doctrine affirmeront que vous êtes pauvres ou malades parce que vous avez péché. Mais dire cela à des Africains équivaut à les tuer. Le Burkina Faso serait-il pauvre parce que nous aurions tous péché? Par contre, si vous êtes en règle avec Dieu, affirment-ils, votre vie sera belle : vous serez riche et vous ne serez jamais malade. La Bible n'a jamais promis ça.Non. Le succès ne prouve jamais qu'on est dans la volonté de Dieu. Une personne peut détourner de l'argent ou le voler, s'enrichir et devenir célèbre. Cela correspond-il au plan de Dieu?

L'adoption d'une vie sainte consiste alors dans le dépassement du mode d'existence vécu par le commun des mortels. Et le témoignage (kaseto) dans «le monde» de leur supplément d'âme s'impose comme un principe d'existence des fidèles. La rigueur observée dans la vie quotidienne des protestants est sous-tendue par la recherche du salut personnel, la certitudo salutis. L'observance de la règle est stricte. Le converti adhère sans réserve à la Bible qui devient son mode d'emploi du monde et sa manière la plus sûre de rentrer en relation avec Dieu (Laurent, 1994).

\section{Modernité insécurisée}

Le second aspect qui caractérise le pentecôtisme du Burkina Faso tient dans la nature particulière de l'Etat qui a finalement favorisé l'apparition d'une forme de modernité globalisée que j'ai qualifiée "d'insécurisée et d'insécurisante ", même si, sur le fond, la modernité comporte toujours une part d'incertitude liée au doute et au relativisme. Il convient aussi de souligner que la croissance récente du Pentecôtisme dans ce pays s'est déroulée dans un contexte où il est historiquement dominé par le catholicisme, ce qui contraste avec les pays d'Afrique anglophone où le protestantisme représente la religion dominante.

Aujourd'hui, avec l'entrée en ville, c'est, pour une majorité de la population, l'accroissement de l'insécurité ou peut-être du sentiment d'insécurité. Toujours est-il que les possibilités d'entrevoir le chemin de la survie à long terme s'amenuisent pour des pans entiers de la population. Le temps de la "modernité insécurisée " conjugue l'affaiblissement de la prise en charge coutumière de la vie commune et les difficultés de l'Etat d'organiser dans la sérénité la survie de certains groupes de sa population (que les ressources de la coutume ont cessé d'assumer).

Pour ne donner qu'un exemple, les dotations financières de l'Etat pour l'enseignement et la santé se réduisent et cèdent le pas aux services privés payants. On observe aussi un accroissement de la corruption (Blundo, 2000 ; Blundo et Olivier de Sardan, 2002), de même que le renforcement des sentiments de peur et de méfiance. Autrement dit, parce qu'ils sont confrontés à une sorte de privatisation de l'Etat, c'est à proprement parler à une transformation du processus de l'identification que sont confrontés certains groupes de population. Elle se concrétise par une altération du rapport des individus à l'entourage, de là à l'émergence d'un puissant sentiment de défiance à l'égard d'autrui, ainsi qu'à un brouillage de la production sociale de la ressemblance et de la différence, que celle-ci soit d'origine coutumière ou produite par la ville.

Ce processus s'accélère à partir de la fin des années quatre-vingts où, réduit à sa portion congrue par les Politiques d'Ajustement Structurel (PAS) et la doctrine de la «bonne 
gouvernance » (démocratisation, multipartisme et décentralisation) (Peemans, Esteves et Laurent, 1996 ; Peemans, 2002), l'Etat post-colonial déliquescent (Olivier de Sardan, 2000) a finalement suscité un climat généralisé d'insécurité.

Ce malaise alimente une sorte de "crise sorcière " où chacun se méfie de tous et renoue avec un principe de violence quotidienne ${ }^{40}$. Ainsi, pour peu qu'il ait vraiment existé, le monopole de la violence (dans le sens de celui de la production de la règle) exercé par l'Etat post-colonial s'estompe. Autrement dit, c'est la fonction de tiers (dans le sens ici du tiers-Etat) qui s'amenuise. Dons, dettes et dépendance de l'entourage restent plus que jamais à l'ordre du jour; autant de pratiques qui se complaisent dans la polysémie et l'ambiguïté, laissant plus encore la porte ouverte à la manipulation magique des forces de l'invisible (métaphore qui renvoie à l'idée d'une économie particulière des relations sociales établies sur la peur, la crainte, la défiance, l'autocensure, la délation, l'allégeance) comme principe de contrôle du lien social et comme mode de gouvernement de la société.

Tout se passe comme si nous vivions un moment particulier où l'accumulation fait peur. D'un côté, à celui qui s'y risque, en raison des jalousies qu'elle suscite et des incertitudes qui pèsent à long terme sur la pérennité des ressources (un contrat de travail ne dure que le temps d'un projet de coopération au développement, par exemple). De l'autre, l'accumulation fait peur à celui qui s'estime injustement écarté des principes de la recherche de la sécurité sociale et économique de la société coutumière par l'égoïsme, selon lui, de celui qui amasse.

Dès lors que l'espoir d'une vie réellement autre à laquelle aspiraient des groupes de populations tentés par le passage du village à la ville et que les principes fondateurs de la société se dérobent, la dépendance mutuelle et le recours potentiel à l'entourage (les parents, voisins, amis ou promotionnaires) demeurent toujours la manière la plus sûre d'assurer sa survie ainsi que celle de sa famille. Ces principes restent donc au cœur de l'accumulation et de la forme de l'identité véhiculée dans une ville émergente du Sahel, à défaut d'une autre forme d'organisation de la sécurité sociale et économique effectivement organisée par l'Etat pour l'ensemble de la population.

La crainte, la jalousie, la peur, mais aussi la défiance et l'autocensure, se sont amplifiées et ont conduit les populations à rechercher une autre protection que celle qu'aurait dû fournir aux citoyens l'Etat de droit, comme, par exemple, la garantie de la paix sociale, l'accès généralisé à l'enseignement et au système de santé, l'assurance-chômage, la pension... En conséquence, à défaut de constater la généralisation de ces principes de sécurité sociale et économique, la population, confrontée à la gestion du lien social, à savoir à la gestion des rapports aux autres, a dû recourir à des solutions accessibles à tous et plausibles à leurs yeux pour prendre en charge l'incertitude liée à la survie et se (re)placer ainsi sous la protection de fétiches et plutôt ici d'un Dieu de puissance. Ceci porte sur la soumission à des principes imaginaires qui participent pleinement, par les sentiments de crainte et de peur, à l'instauration d'un processus de dépendance mutuelle et au maintien d'une forme d'économie symbolique alimentée par les dons, l'accumulation de dettes et le recours, si nécessaire, à la manipulation des forces de l'invisible. Autrement dit, pour le dire métaphoriquement, une sorte de pensée magique appartient à cette forme de la modernité.

31 Dans la "modernité globalisée » de cette région, tout se passe comme si un principe d'individualisme - qui n'implique donc pas ici le désassujettissement des hommes vis-àvis des dieux, dans le sens ici de la production imaginaire des principes de pouvoir parvenait à composer avec le collectif, soit avec l'univers de la dépendance de l'entourage 
(comme garantie de la meilleure sécurité sociale et économique pour soi et sa famille), ceci non sans raviver la peur, la violence, la jalousie, la rancœur, la vengeance, l'allégeance qui saturent, plus encore, les relations humaines et suggèrent l'idée d'une résurgence des "pouvoirs sorciers» (Tonda, 2003; Bernault et Tonda, 2000). En conséquence, nous sommes tenus de prendre en considération un processus singulier de production de l'identité citoyenne : dans ce climat de suspicion qui sape la confiance dans la parole de l'autre, c'est l'établissement d'un lien social pacifié qui paraît parfois vaciller.

Autrement dit, c'est la violence que je peux mobiliser à titre personnel qui permet d'instaurer la relation à l'autre. En conséquence, les rapports sociaux restent essentiellement magiques (dans le sens d'une magie travaillée par l'entrée dans cette forme de modernité tardive), régulés par le truchement de la manipulation de forces invisibles qu'exprime l'émergence, sans précédent, d'une forme de sorcellerie, inédite à ce jour, et le développement de mouvements religieux. Si on ne sort donc pas vraiment du paradigme de la puissance où "force et immanence définissent la religion païenne » (Augé, 1982), il s'agira néanmoins de rendre compte des principes de transformation d'une pensée magique - soit celle d'une humanité qui entretient des rapports oblatifs avec les esprits (De Heusch, 1986) -, religieuse, et une conception de la personne basée sur un « moi » composé de plusieurs instances (Bastide, 1973).

Dans cet environnement, les Eglises des AD peuvent être comprises, pour les membres de la communauté exclusivement, comme un lieu d'apaisement face à la gestion magique (où règne la peur, la défiance, la rancœur) des rapports entre les individus. L'adhésion aux principes éthiques formulés par la Bible et la participation aux rituels de guérison animés par les croyants-guérisseurs, par exemple, peuvent être interprétées comme la recherche d'un espace où s'invente et s'expérimente concrètement la confiance rétablie entre «frères et sœurs en prière » face à l'univers de défiance qui sévirait à l'extérieur. En d'autres termes encore, les fidèles rassemblés autour de leur pasteur constituent une "famille de prière " au sein de laquelle chacun peut "déposer son fardeaux »- soit le poids des jalousies, des peurs et des rancœurs ressenties - afin de renouer avec la confiance dans un entourage par la possibilité de réaliser des échanges de dons (dont les formes d'alliance) et de s'endetter.

\section{A propos de la problématique centrale du livre}

Joël Noret ne discernant pas le rapport dynamique entre "pentecôtisation » et société burkinabé qu'il confond avec ce qu'il appelle le contexte social "local», ignore en conséquence la problématique centrale de mon travail. Ce faisant, il me donne l'opportunité de préciser certains aspects de mes travaux à la faveur desquels je répondrai aux observations et autres questions qui m'ont été adressées.

\section{« Concorde coutumière » et « concorde civile », « 'moi' multiple »}

C'est un truisme de rappeler que la distinction entre "concorde coutumière » et " concorde civile» fut établie par souci de schématisation et donc de clarification de l'exposé. Ce principe méthodologique m'a permis d'énoncer plus clairement les termes d'un débat complexe. Dans le contexte contemporain du plateau mossi du Burkina Faso et plus particulièrement dans le cadre des petites villes émergentes, j'ai suggéré que nous pourrions être en présence d'un modèle impur d'accord entre les groupes de personnes, 
modèle qui tente de s'établir sur un principe d'ajustement situé entre, au-delà ou à côté des principes de la « concorde coutumière » et ceux de la « concorde civile». Autrement dit, comment les groupes de populations parviennent à partiellement dépasser, ou plutôt, à composer avec « l'empilement normatif » producteur d'insécurité (pour les plus faibles) en raison de la compétition entre différents systèmes hiérarchiques pour forcer, malgré tout, un type d'accord (singulier) entre les parties? ${ }^{41}$ Ce principe de «bi-localisation » des formes de régulation de la vie en commun, entre le «lieu local » et le «lieu citadin", trouve son expression dans une sorte de «conversion bien tempérée » qui se situe entre des offres plurielles de citoyenneté, avec l'espoir d'édifier une synthèse inédite entre ces modes d'existence, et des principes de la sécurité sociale et économique, a priori incompatibles.

C'est donc pour traiter en amont des éléments qui informent sur cette situation composite qu'il m'a semblé utile de réserver l'utilisation de la notion de «concorde civile » à l'instauration des conditions d'une accumulation pour soi et ses proches, soit une situation où la satisfaction de l'intérêt individuel, étendu au plus grand nombre de personnes, implique l'édification d'un espace public. Autrement dit, les relations sociales s'établissent ici sur un état de confiance, généralisé à tous les membres de la société, confiance qui implique l'existence d'un ensemble complexe de règles reconnues par tous, garanties par une instance supérieure, tierce, l'Etat de droit en l'occurrence. La « concorde civile» conduit à la production de citoyens dotés de droits et de devoirs; il s'agit d'individus gratifiés d'une responsabilité pleine et entière. Ceci diffère de la situation rencontrée dans la société coutumière mossi, où la confiance (kis-sida) est essentiellement réservée aux relations de parenté et l'entente (wuum taaba) - qui ne peut pas être confondue avec la kis-sida, la confiance -, est instaurée entre les différents lignages qui constituent la société.

Dès lors, pour être en mesure de vider la querelle et de pouvoir distinguer publiquement le vrai du faux ou le bien du mal, et donc pour que s'instaurent la " concorde civile » et l'espace public afférent, il faudrait, d'une certaine manière, être définitivement quitte de l'autre, dans le sens ici d'être capable de s'émanciper de l'économie de l'affection (l'univers de la dépendance). Alors que, dans le contexte qui nous retient, le don et la dette induisent la dépendance mutuelle et donc des réseaux d'affinités électives qui participent à la constitution d'un principe de sécurité pour soi et ses proches. Autrement dit encore, l'espace public de la "concorde civile», pour s'imposer, nécessite "l'émancipation de la parenté » et/ou de la logique des réseaux (personnalisation des relations, ce qui renvoie à l'idée de privatisation de l'Etat (Medard, 1992 ; Jaffre et Olivier de Sardan, 2003), logique qui diffère de l'anonymat du traitement des individus dans leurs rapports à une administration publique. Il en résulte que la «concorde coutumière » et les espaces collectifs qui lui sont propres privilégient l'entente pour la société et donc évitent par dessus tout, la mise au jour des conflits. La « concorde coutumière » repose pour une large part sur l'imposition d'un consensus par des personnages notoires de la société. Elle renvoie aussi à une conception de l'unité de la personne basée sur une pluralité des instances, dans le sens d'une structure relationnelle entre des principes vitaux intérieurs et extérieurs (Bastide, 1973; Godelier et Panoff, 1998; Taylor, 1998) ${ }^{42}$, conception que j'ai présentée schématiquement en reprenant la formule de Mary Douglas $\mathrm{du}$ « moi » multiple dans un seul corps, avec l'intention de focaliser l'attention sur les conséquences déterminantes de cette conception sur la vie en commun et sur l'idée de responsabilité personnelle (Douglas, 1999: 160) ${ }^{43}$. On le comprend, au-delà de cette 
dichotomie, mon objectif général consiste à rendre compte de l'imaginaire diabolique des pentecôtistes mossi, sachant que notre culture occidentale tient du point de vue de la rationalité le monde des «forces de l'invisible » comme de la pure imagination. Il fallait donc me démarquer d'une conception qui m'aurait conduit à une forme d'évolutionnisme politique où l'espace public, considéré comme le lieu par excellence du politique, rime avec la transparence, afin de considérer la sorcellerie comme un autre discours sur le pouvoir politique et la richesse.

\section{Confiance}

Dans ce débat, la problématique de la confiance est déterminante et la description de sa variabilité de nature entre la "concorde coutumière " et la "concorde civile", en relation à la société burkinabé, attira toute mon attention. Ainsi, avec l'Etat-nation, les relations de confiance se généralisent à tous les citoyens, à la différence de la plupart des sociétés coutumières où la confiance entre les personnes est réservée essentiellement aux relations de parenté. Aujourd'hui, là où l'Etat-nation a pu légitimement s'imposer, c'est la responsabilité de l'humanisation en oeuvre au sein de la famille (c'est-à-dire l'alliance qui, dans le sens lévi-straussien, institue la paix, la coopération et la société) qui lui est transférée. L'Etat assume dans ce cas le rôle de tiers, soit de garant de l'impartialité et de la vigilance, dès lors qu'il est défini par son obligation première d'assurer la sécurité de quiconque réside sur son territoire délimité par des règles institutionnelles qui légitiment et obligent cet Etat (Ricoeur, 2000 : 432). De ceci, il devient claire que cette partie de l'ouvrage consiste en une modeste contribution à la description d'un état de confiance établi par des groupes de population, lorsque celui-ci résulte de la coexistence de différentes réalités historiques, dans un même présent, moment que j'ai qualifié (à défaut de mieux) de «modernité insécurisée et insécurisante » dans le sens où la confiance n'est ici ni vraiment produite par les relations de parenté, ni par les institutions étatiques. Dès lors que devient la nature du lien social? Et comment peut-on décrire la construction de l'identité?

\section{Identité : rupture et bricolage social}

39 A ce propos, si les remarques de Joël Noret sont stimulantes, elles sous-évaluent les difficultés conceptuelles soulevées par le terrain. Dans l'état actuel du débat, j'ai cherché à les énoncer de manière logique par un recours aussi précis que possible à la description ethnologique ${ }^{44}$.

En bref et schématiquement ici, j'ai montré que pour le pentecôtisme historique, la conversion équivaut généralement à une rupture radicale. Les fidèles des $\mathrm{AD}$ ne négocient pas avec la coutume, ils la combattent. Dans ce sens, je reste à ce niveau wébérien ( $c f$. ma problématique du " groupe de la sortie du groupe »45. Cette rupture oppose la conception d'une vie éternelle et d'un paradis duquel on ne revient plus sur terre, aux croyances coutumières selon lesquelles il n'y a pas d'être neuf mais plutôt les mêmes personnes qui reviennent de "l'autre monde", dans le monde des vivants (cf. supra: la conception de la personne). Les conséquences sociologiques sont considérables: ce qui est désormais promis, c'est d'être quitte de l'âpreté de la survie en milieu rural et le salut devient la vie éternelle. Dans cette vision, le paysan est celui qui ne possède rien et, dans ce sens, il découvre la pauvreté. Autrement dit, il s'agit du rejet de l'insupportable de la condition 
paysanne telle qu'elle apparaît désormais aux fidèles des $\mathrm{AD}$ (pénibilité, misère, non accès aux écoles et aux soins médicaux...), et la figure du diable s'accroche à la peur de trop bien se reconnaitre dans l'image de ce paysan.

En milieu mossi, le génie du pentecôtisme consiste à permettre aux fidèles de sauvegarder les acquis de la conversion (qui portent sur l'adoption d'une autre identité et l'établissement de nouvelles relations à l'entourage), sans les compromettre dans les inévitables négociations engendrées par la dureté des conditions d'existence. Par cette subtile distinction, nous sommes en présence d'un bricolage qui ne se considérant absolument pas comme tel parce qu'il porte précisément sur une institution sociale (le mariage par exemple), est toléré. Ou pour mieux dire, ce bricolage permet de maintenir intact la rupture liée à la conversion. A titre d'exemple, j'ai montré que les formes subtiles d'alliances inventées par les $\mathrm{AD}$ (j'ai parlé à ce propos d'un bricolage social qui instrumentalise les alliances coutumières après les avoir « découtumisées ») préserve le recours à l'entourage et donc partiellement la sécurité par la communauté, tout en rejetant catégoriquement toute idée de bricolage culturel ${ }^{46}$. La distinction entre un bricolage social et un éventuel bricolage culturel s'imposait donc. Le pentecôtisme historique reste avant tout une rupture qui ne laisse pas de place à ce niveau à la coexistence ; il ne s'agit donc pas du principe de coupure bien connu et décrit par Roger Bastide ${ }^{47}$ pour d'autres lieux, soit d'une capacité métaphysique à vivre dans deux mondes, sans en ressentir les contradictions (ni d'ailleurs de la situation de coexistence des contraires décrite pas P. Bourdieu et A. Sayad). Il n'y a pas ici de double entente, mais un déclassement radical de la coutume, annulation de toute référence aux ancêtres par exemple, afin d'en récupérer d'autant mieux et de manière pragmatique certaines institutions sociales indispensables à la survie.

Plus précisément, le principe de coupure chez Roger Bastide rend compte de manière féconde d'un mécanisme de défense de groupes minoritaires, et plus particulièrement, dans le cas étudié par l'auteur, celui composé d'esclaves noirs et de leurs descendants de l'époque de l'Etat colonial portugais à celle de l'Etat brésilien. Son ouvrage majeur, Les religions africaines au Brésil, est traversé d'une distinction (articulation) entre société et civilisation, rites et mythes (à propos de la mémoire collective, Bastide, $1960: 335$ ), ou encore, entre les "structures sociales brisées" des esclaves noirs et leurs "valeurs conservées» (Bastide, 1960 : 78-79, 92). Ma distinction entre bricolage social et bricolage culturel est formellement assez proche, dans la mesure où mon intention vise à intégrer à l'analyse le contexte socio-économique. Toutefois, en raison de la spécificité sociohistorique de mon terrain, j'aboutis à la conclusion inverse de Bastide. Ainsi, là où les valeurs sont conservées, pour les esclaves noirs, les pentecôtistes du Burkina Faso déclarent obsolètes les valeurs de la société traditionnelle ( $c$ f. la métaphore de la « vieille peau » dont ils veulent se débarrasser) et là où « les structures sociales étaient brisées " pour les esclaves noirs au Brésil déracinés de leurs sociétés africaines d'origine, les structures sociales de la société mossi sont maintenues, certes avec des transformations, en raison essentiellement d'impératifs liés aux difficultés matérielles des fidèles ( $c f$. le bricolage de l'institution sociale du mariage). Le principe de coupure s'oppose donc à celui de rupture; le premier porte sur la résistance (Bastide, 1960:362) et la double fidélité (Bastide, 1960 : 98). Bastide parle à ce propos de ruses (Bastide, 1960 : 67, 95, 159, $375,380 \ldots$...) et de masques (Bastide, $1960: 67,225$ ) pour rendre compte des relations entre les esclaves et les maîtres blancs, tandis que la notion de rupture traite surtout d'adhésion et de conversion : les fidèles mossi n'évoquent-ils pas l'idée d'une « conversion 
de cœur » et non "de bouche ", ou encore « de se donner complètement à Jésus »? Ceci renvoie à une adhésion pleine et entière à une forme de la modernité et non à une résistance à son égard (Tonda, 2003 : 137, 237-239). Dans ce but, les fidèles mossi bricolent avec le rituel du mariage à la condition sine qua non de l'avoir préalablement "découtumisé » (démystifié d'une certaine manière). La rupture est reliée à ma problématique, à l'accent wébérien, « du groupe de la sortie du groupe » et donc aussi à la conversion et au doute (cf. mon analyse de l'offre de guérison des $\mathrm{AD} / \mathrm{BF}$ où le conflit intérieur non résolu lié à la rupture engendrée de la conversion rend malades certains fidèles, ce qui diffère de la simultanéité de comportements différents sans conflit intérieur qui se trouve au cœur du principe de coupure) ; le principe de coupure témoigne plutôt de la sauvegarde de la culture native (de ses valeurs), il y a alors ruse et résistance face aux dominants ${ }^{48}$, ce que Bastide montre lorsqu'il décrit, par exemple, le marronnage.

Bien plus, le radicalisme de la conversion permet au fidèle d'adopter d'une vision essentiellement dualiste du monde qui le pousse à transformer sa perception des relations aux autres. Il s'agit donc bien de traiter de la construction de la catégorie du mal. En deux mots, j'en ai fait longuement état dans le livre, la société coutumière, défaite, équivaut désormais à un principe démoniaque, vis-à-vis duquel il convient d'avoir l'audace de se distancier, au même titre que de «la société (moderne)» identifiée « au monde ». En référence à la Bible, qui crée "un monde», les protestants peuvent désormais décoder les rapports sociaux en termes de bien et de mal. De sorte que les convertis peuvent se mouvoir dans la noirceur «du monde", dotés qu'ils sont de la capacité de discerner le bien (le respect des principes bibliques et, par delà, l'instauration de relations avec autrui basées sur la confiance, la justice, le pardon), du mal (le démon et les pratiques qui y renvoient). Dans ce sens, le strict respect des interdits religieux revêt une importance particulière, car il produit chez les convertis le sentiment d'appartenir à une communauté transnationale qui leur offre une ouverture sur le monde que la modernité promet (voir ci-dessus).

\section{Identité : guérison et bricolage culturel}

44 C'est seulement dans un deuxième temps, lorsqu'on tente de relier la question de l'identité à la guérison divine, que les choses se compliquent singulièrement. Il s'agit alors de traiter d'un pentecôtisme de "seconde génération ", incarné, entre autres, par les croyants guérisseurs, véritables passeurs de frontières, qui exercent généralement leur art à la lisière de la doxa pentecôtiste. A la situation précédente du « groupe de la sortie du groupe » se surajoute un autre moment de réforme importante de la culture, lié à la modernité globalisée, à l'urbanisation rapide du Sahel et à l'affaiblissement de l'Etat postcolonial. J'ai montré que ces changements alimentent ici une sorte de « crise sorcière » où chacun se méfie de tous. Et à défaut d'un mécanisme de régulation de la violence inhérente à la vie en commun, la conduite de la collectivité s'exprime dans un face-à-face permanent entre les individus, où les confrontations entre leurs « puissances » (dont les fétiches) offensives ou protectrices deviennent la norme. Dans ce contexte, au delà de la référence à $\mathrm{B}$. Lahire ${ }^{49}$, c'est vers une sociologie centrée sur le sujet, ainsi que sur les travaux qui traitent d'une identité en rupture avec les institutions qu'il me semble intéressant de se tourner ${ }^{50}$. Toutefois, le problème crucial, voire insurmontable, deviendrait alors celui de la pertinence d'une comparaison entre des univers globalisés certes, mais distants. Ainsi par exemple, et là réside le cœur de la problématique esquissée ci-dessus, cette « crise sorcière » conduit à l'idée de la manipulation magique 
des forces de l'invisible comme principe de l'établissement du lien social et comme mode de gouvernement de la société: "Tout le problème est que la sorcellerie relève fondamentalement de l'univers du soupçon et de la rumeur: le sorcier, le diable, c'est l'autre qui vous jalouse... Pour sortir des impasses d'un fonctionnement 'à l'imaginaire' où le sorcier et l'ensorcelé (...) se renvoient la balle, les sociétés traditionnelles ont toujours eu recours à des médiations symboliques et à des procédures d'ordalie» (Corten et Mary, 2000). C'est dans ces circonstances qu'on retrouve le «moi» multiple qui certes par rapport à sa conception traditionnelle, a subi quelques transformations ${ }^{51}$.

Dans ce contexte, j'ai montré que la maladie renvoie, pour partie, à un mal-être, à savoir à des «pathologies de l'entre soi ». Le traitement consiste généralement dans la mise à bonne distance de l'entourage par l'entretien d'une tension entre affiliation et désaffiliation. Par l'exorcisme, les patients expriment leurs doutes, ceux qui les ont conduits à la remise en question de la rupture radicale d'avec "l'ancien monde ». Les croyants guérisseurs viennent en quelque sorte prendre en charge cette indécision, considérée ici comme l'élément pathogène à la source de la souffrance. Elle résulte d'un choix à faire, toujours différé, entre les principes incompatibles de la vie en commun de la société coutumière et ceux inhérents à la vie sociale de l'individu sujet de la modernité. Avec l'offre de guérison, la simple « ruse » du bricolage social (cf. les formes du mariage chez les $\mathrm{AD}$ ) ne suffit plus. Il faut alors mobiliser, ponctuellement, le temps d'apaiser le doute, une sorte de bricolage culturel avec l'espoir d'édifier une synthèse inédite entre des modes d'existence et des représentations du monde a priori incompatibles ${ }^{52}$. Mais ceci est inavouable et dans ce cas, le travail thérapeutique peut être identifié à une forme d'hybridation culturelle qui doit cependant rester discrète, voire secrète car le pentecôtisme se vit et s'affiche avant tout comme une rupture ne tolérant aucune faiblesse. Le bricolage culturel est ici le fruit d'une souffrance ; il renvoie en quelque sorte à l'aveu de la défaite du fidèle qui hésite et donc doute. Cette défaite peut aussi être vécue comme une sorte d'arrêt dans la quête (la croyance) d'une marche en avant vers la modernité (Tonda, 2003 : 237) incarnée par la conversion (la rupture).

C'est seulement à la faveur de la consultation privée du croyant guérisseur, en marge de la doctrine des $\mathrm{AD}$, que ce doute trouvera un écho, tandis que l'autre scène de la guérison - inséparable de la première -, celle officielle de la prière collective, se concentre sur la toute-puissance de Dieu et le combat victorieux contre le mal. La guérison est la découverte de la conversion pour certains et l'expérience forte d'une "reconversion " pour d'autres, soit une réaffirmation de la certitude de la rupture; pour vivre dans la plénitude, répètent inlassablement les pasteurs, il faut «se convertir de cœur» et pas « de bouche ». C'est seulement à la condition de reconnaître cette scène thérapeutique duale, où se joue alternativement le doute (oscillation) et la rupture, que les $\mathrm{AD}$ deviennent un lieu où « déposer son fardeau " ${ }^{53}$, soit le poids des jalousies, des peurs et des rancœurs ressenties. Les méandres de l'étiologie de la consultation privée du croyant guérisseur, au-delà de la rhétorique du mal absolu, celle du diable, recourent aux " mauvais esprits »- aux zinse (sing. zina ${ }^{54}$, ancêtres errants issus d'une mauvaise mort), mais surtout aux génies de l'adultère, de la colère, de la paresse, ou encore de l'alcool - lesquels profiteraient de la faiblesse d'un individu pour « entrer » en lui (cf. dans le livre, les cas du "vieux à la charrette », du pasteur Jonas, ou celui de l'exorcisme d'une jeune fille par un croyant guérisseur) ${ }^{55}$. Autrement dit, pour reprendre les catégories des pentecôtistes mossi, «se dire diable » ou encore «être déclaré diable » (possédé), c'est aussi ne pas vraiment se sentir coupable ni responsable (Zempleni, 1975: 167). Ce 
principe explicatif, en marge de la doxa, renvoie à une pensée syncrétique qui combine un principe de persécution (untel m'a envoyé tel génie) ou une cause individuelle (un écart à la Loi) avec la conception d'un « moi » multiple en mesure de disculper le fidèle (ce n'est pas moi le coupable mais l'esprit d'adultère...). Dans ces « disculpations douces comme dans ces fictions polies» (Douglas, 1999: 160), ce qui compte en définitive, c'est de parvenir à accuser tout en gardant intact son capital social comme un recours dans l'adversité ; celui qu'il faut ménager, c'est bien l'autre qui vous persécute et que vous accusez. Autrement dit, la communauté et les principes identitaires qui lui sont propres, tout en se transformant, parviennent à instaurer des relations avec l'individu sujet de cette forme de modernités ${ }^{5}$.

\section{Corps en souffrance}

47 La scène thérapeutique des $\mathrm{AD}$ est un lieu de tensions parfois extrêmes pour les malades et la remarque de Sandra Fancello concernant ma faible insistance sur la violence du corps à corps fidèle-guérisseur est sûrement fondée bien que je ne l'aie pas ignorée. Ainsi, lorsque je dépeins par exemple le croyant guérisseur Elisée comme «le boxeur de la foi », ou encore lorsque je rends compte d'une séance de délivrance ${ }^{57}$ : «(...) Il s'accroupit devant une femme et, le doigt pointé vers sa bouche, somme les démons de fuir. Il répète rageusement la même phrase : 'Démon, ramasse tes affaires ou je te brûle!'. Le démon résiste. La femme malgré ses liens se tord. Dans un rythme très soutenu, il insiste : 'Sors, sors, sors, sors, sors, ...'. Elle pousse des cris étouffés. Ses yeux sont révulsés, sa respiration est rapide et sa bouche produit une abondante écume. Deux pasteurs prennent le relais du croyant-guérisseur et, lorsque la patiente semble perdre connaissance et son corps se détendre, ils l'abandonnent pour poursuivre ailleurs le combat... ». Toutefois, il est vrai que ces descriptions restent en deçà de la réalité. Je n'ai vraiment pris la mesure de la gestuelle du corps dans l'expression parfois extrême de la souffrance et dans le travail des croyants guérisseurs qu'après avoir visionné le film que j'ai tourné en décembre 2002 à l'occasion d'une séance de délivrance. On y voit, à la limite du soutenable, des corps en souffrance qui expriment un mal, autrement innommable, lié au doute, à la peur, au malheur, soit le plus souvent l'atermoiement devant la défaite de l'ancien monde et les difficultés de la survie. Ces instants sont aussi ceux, pour certains, de la remémoration de la conversion, souvenir intensément vécu comme un moment dramatique d'abandon de soi, suite à la prise de conscience de la défaite des anciennes puissances protectrices. Et la foi est aussi l'expérience vraie d'une émotion collective liée aux manifestations du Saint-Esprit que vivent concrètement les fidèles, par un engagement physique total. Les cris, les pleurs, les tremblements expriment l'angoisse face au vide d'un devenir entrevu, et que ne comble pas l'espoir d'un monde nouveau annoncé mais encore inconnu et que prendra en charge la posture thérapeutique duale des AD. 


\section{BIBLIOGRAPHIE}

AUGÉ, M., 1982, Génie du paganisme, Paris : Gallimard.

BAJoIT, G., 2003, Le changement social. Approche sociologique des sociétés contemporaines, Paris : Armand Colin.

BALANDIER, $G$.

1955a, Sociologie actuelle de l'Afrique noire, Paris : P.U.F.

1995b, Préface. « Une sociologie de la rencontre », in Bastide R., Les religions africaines au Brésil, Paris : PUF, pp. V-IX.

BASTIAN, J.-P. (éd.), 2001, La modernité religieuse en perspective comparée. Europe latine -

Amérique latine, Paris : Karthala.

BASTIDE, R.

1960, Les Religions africaines au Brésil, Paris : P.U.F. (seconde édition 1995).

1973, « Le principe d'individuation (contribution à une philosophie africaine) », in La notion de personne en Afrique noire, Colloques Internationaux du CNRS, n 544, Paris, l'Harmattan, pp. 33-43.

BERnAult, F. et TONDA, J. (éds.), 2000, « Pouvoirs sorciers (dossier) », Politique africaine, 79.

BLUNDO, G. (éd.), 2000, « Monnayer les pouvoirs. Espaces, mécanismes et représentations de la corruption », Nouveaux cahiers de l'IUED, 9.

BLUNDO, G. et OLIVIER DE SARDAN, J.-P. (éds.), 2002, « La corruption en question », Politique Africaine, 83.

BONNET, D., 1988, Corps biologique, Corps social. Procréation et maladies de l'enfant en pays mossi. Burkina Faso, Paris, ORSTOM, Mémoires nº 110.

COMAROFF, J. et J., 2000, « Réflexions sur la jeunesse. Du passé à la postcolonie », Politique africaine, 80, pp. 90-110.

CORTEN, A., 1995, Le pentecôtisme au Brésil. Emotion du pauvre et romantisme théologique, Paris : Karthala.

CORTen, A. et MARShall-Fratani, R. (éds.), 2001, Between Babel and Pentecost : Transnational Pente-costalism in Africa and Latin America, London : Hurst.

CORTEN, A. et MARY, A., 2000, Imaginaires politiques et pentecôtistes. Afrique/Amérique latine, Paris : Karthala.

DE HEUSCH, L., 1986, Le sacrifice dans les religions africaines, Paris : Gallimard.

DE SURGY, A., 2001, Le phénomène pentecôtiste en Afrique noire. Le cas béninois, Paris :

L'Harmattan.

DOUGLAS, M.

1971, De la souillure. Essais sur la notions de pollution et de tabou, Paris : Maspero.

1999, Comment pensent les institutions, Paris : La Découverte / MAUSS. 
Dozon, J.-P., 1985, La société bété. Côte-d'Ivoire, Paris : Karthala.

DUBAR, C., 2000, La crise des identités. L'interprétation d'une mutation, Paris : PUF.

DUBET, F., 1994, Sociologie de l'expérience, Paris : Seuil.

FIALHO-COSTA, L., 2002, Qu'est-ce qui fait crier les crentes? Emotion, corps et délivrance à l'Eglise Universelle du Royaume de Dieu (Bahia-Brésil), Paris, EHESS, thèse de doctorat.

GARCIA-CANCLINI, N., 1995, Culturas híbridas. Estrategias para entrar y salir de la modernidad, Buenos Aires : Editorial Sudamericana, 2ème édition.

GEERTZ, C.

1973, The Interpretation of Cultures, New York : Basic Books.

1986, Savoir local, savoir global. Les lieux du savoir (trad. Denise Paulme), Paris : PUF.

Godelier, M. et Panoff, M., 1998, La production du corps, Paris : Ed. des archives contemporaines. JAFFRE, Y. et Olivier de Sardan, J.-P. (éds),

2002, Les dysfonctionnements des systèmes de soins. Rapport du volet socio-anthropologique. Enquêtes sur l'accès aux soins dans cinq capitales d'Afrique de l'Ouest, Projet "Santé urbaine », UNICEF, Coopération française.

2003, Une médecine inhospitalière. Les difficiles relations entre soignants et soignés dans cinq capitales d'Afrique de l'Ouest, Paris : APAD-Karthala.

LAURENT, P.-J.

1994, « Prosélytisme religieux, intensification agricole et organisation paysanne. Le rôle des AD dans l'émergence de la Fédération Wend-Yam au Burkina Faso », in Jacob, J.-P. et Lavigne Delville, P., Les associations paysannes, organisation et dynamiques, Paris : APAD-IUED-Karthala, pp. 155-179.

1998a, « Conversions aux Assemblées de Dieu du Burkina Faso : modernité et socialité », Journal des Africanistes, tome 68, 1-2, pp. 67-98.

1998b, Une association de développement en pays mossi. Le don comme ruse, Paris : Karthala.

1999, «L'Eglise des Assemblées de Dieu du Burkina Faso : histoire, transitions et recompositions identitaires ", Archives des sciences sociales des religions, 105, pp. 71-97.

2002, «Effervescence religieuse et gouvernance. L'exemple des Assemblées de Dieu du Burkina Faso », Politique africaine, 85, pp. 95-116.

2003a, Les pentecôtistes du Burkina Faso. Mariage, pouvoir et guérison, Paris : IRD-Karthala.

2003b, Aspects du processus d'expansion du pentecôtisme en Afrique de l'Ouest. L'exemple du travail missionnaire des Assemblées de Dieu du Burkina Faso, (séminaire IFRAN - oct. 2003), Paris : Karthala (à paraître), $23 \mathrm{p}$.

LAURENT, P.-J. et MARY, A. (éds.), 2001, « Prophètes visionnaires et guérisseurs de l'Afrique subsaharienne contemporaine », Social Compass, Sage, vol. 48, 3, sep. 2001, pp. 307-480.

LEVI-STRAUSS, C.

1958, Anthropologie structurale, t. 1, Paris : Plon.

1962, La pensée sauvage, Paris : Plon.

1964, Le cru et le cuit, Paris : Plon.

1985, La potière jalouse, Paris : Plon. 
LUCA, N., 1999, « Pentecôtisme en Corée », Archives de Science Sociale des Religions, 105, pp. 99-123.

MARWICK, M., 1965, Sorcery in Its Social Setting. A Study of the Morthern Rhodesian Cewa, Manchester : Manchester University Press.

MARY, A.

1999, Le défi du syncrétisme, Le travail symbolique de la religion d'Eboga (Gabon), Paris : EHESS.

2000, Le bricolage africain des héros chrétiens, Paris : CERF.

MEBToul, M., 2002, « Capital relationnel et logiques d'acteurs dans les services de santé : Algérie », Leiden, Colloque APAD, 22-25 mai 2002..

MEDARD, J.-F., 1992, « Le big man en Afrique. Du politicien entrepreneur », L’Année sociologique, 42, pp. 167-192.

NASCIMENTO, E. L., 1995, « Praise the Lord and Pass the Cath-up », News from Brasil, Cover story. OLIVIER DE SARDAN, J.-P., 2000, « Dramatique déliquescence des Etats en Afrique », Le Monde Diplomatique, février 2000, pp. 12-13.

ORTIGUES, M.-C. et E., 1984, Oedipe africain, Paris : L'Harmattan.

PEEL, J. D. Y., 1968, « Syncretism and religious change », Studies in Society and History, 10, pp.

121-141.

PEEMANS, J.-P., 2002, Le développement des peuples face à la modernisation du monde, Louvainla-Neuve/Paris : Academia-Bruylant/L'Harmattan.

PeEMANS, P.-J., ESTEVEs, A. et LAURENT, P.-J., 1996, Stabilité politique, ethnicité et dimensions socioéconomiques de la gouvernementalité locale, Bruxelles : AGCD.

PIAULT, C. (éd.), 1975, Prophétisme et thérapeutique, Paris : Hermann.

PITT-RIVERS, J., 1997, Anthropologie de l'honneur, Paris : Hachette.

POULET, E., 1969, Contribution à l'étude des composantes de la personne humaine chez les Mossi, Thèse de doctorat de troisième cycle, Université de Clermont-Ferrand.

RAMIREZ, R., 1999, What It Means to Be a Man, New Brunswick, New Jersey : Rutgers University Press.

RICOEUR, P.

1960, Finitude et culpabilité, II. La symbolique du mal, Paris : Aubier.

2000, La mémoire, l'histoire, l'oubli, Paris : Seuil.

TAYLOR, A.-C., 1998, « Corps immortels, devoir d'oubli : formes humaines et trajectoires de vie chez les Achuar », in Godelier, M. et Panoff, M. (éds.), La production du corps, Paris : Editions des archives contemporaines, pp. 317-338.

TONDA, J., 2002, La guérison divine en Afrique centrale (Congo, Gabon), Paris : Karthala.

WILLAIME, J.-P. (éd.), 1999, « Le pentecôtisme : les paradoxes d'une religion transnationale de l'émotion », Archives des sciences sociales des religions, 105.

ZEMPLENI, A., 1975, « De la persécution à la culpabilité », in Piault, C. (éd.), Prophétisme et thérapeutique, Paris : Hermann, pp. 153-218. 
ZUBILLAGA, V., 2003, Entre hombres y culebras. Devenir un homme et se faire respecter dans un barrio d'une ville latino-américaine, Louvain-la-Neuve, Thèse de doctorat.

\section{Production des Assemblées de Dieu}

75 ${ }^{2 m e}$ anniversaire des Assemblées de Dieu du Burkina Faso 1921-1996, 1996, 96 p.

Annuaire Evangélique du Burkina Faso 2002-2003, 104 p.

Contact, magazine lié à la mouvance évangélique du Burkina Faso, un numéro par an.

Flamme, magazine officielle des Assemblées de Dieu du Burkina Faso (depuis 1994, quatre numéros par an).

Pasteur Jean Pawentaoré Ouedraogo, s.d., Aperçu sur l'histoire des Assemblées de Dieu en Côte d'Ivoire, $14 \mathrm{p}$.

« Where we've been », A/G online USA (site officiel des Assemblées de Dieu).

\section{NOTES}

1. Cette question renvoie à la discussion du concept de « variation d'échelle » (Ricoeur, $2000: 273$, 276), voir aussi Geertz (1973 et 1986).

2. Croyants-guérisseurs wend nor ressa (l'interprète de Dieu) ou wend nam tem tuumd da (celui qui fait le travail de Dieu), fidèles baptisés de l'Esprit Saint et qui possèdent, entre d'autres dons, celui de guérison, les croyants-guérisseurs se situent le plus souvent, à la lisière de la doxa pentecôtiste.

3. Les catégories du bien et du mal sont à distinguer de l'idéologie de l'entente (wuum taaba, soit aussi, le consensus, l'arrangement), qui vient réguler les relations entre les multiples composantes de la société mossi et implique plutôt la prudence, la discrétion, le respect, le secret, la ruse, la polysémie, et la crainte (pour de plus amples informations : Laurent, 1998b).

4. Ces aspects renvoient au débat majeur concernant les transformations des structures. $C f$. les travaux de Lévi-Strauss (1958, 1962, 1964, 1985) ; voir aussi : Bastide (1960), Balandier (1971), Augé (1982), Peel (1968) et Mary (2000).

5. Pour plus d'informations sur ces questions : Laurent (2003b).

6. J'ai retenu l'estimation la plus basse. Données fournies en 2002 par la Fédération des Eglises et Missions Evangéliques (FEME) qui regroupe 4 missions et 17 Eglises locales.

7. Chiffre fourni par la Mission des $\mathrm{AD}$ de France à Ouagadougou (siège de Tanghin barrage) ; pour sa part, l'Annuaire Evangélique 2002-2003 (p. 13) parle de 3.000 églises des AD.

8. Chiffre fourni par la Mission des AD de France à Ouagadougou (de Tanghin barrage). Actuellement les 6 écoles bibliques (sans compter l'Institut supérieur de théologie) forment entre 150 et 200 jeunes pasteurs par an (formation de trois ans) (Flamme, 18, 1999, p. 12).

9. Selon les statistiques qui m'ont été fournies par les responsables des AD en avril 2003 (voir aussi, Annuaire Evangélique 2002-2003, p. 13). En 1972, l'Eglise des AD de Haute-Volta fête son cinquantième anniversaire. Selon leurs propres statistiques, l'Eglise comptait 125.000 fidèles, essentiellement répartis en milieu rural et dans les petits centres urbains, sur une population du pays estimée à un peu plus de 5 millions d'habitants, soit 2,5\% de la population totale. En 1996, à l'occasion de leur $75^{\mathrm{e}}$ anniversaire, les $\mathrm{AD}$ rassemblaient près de 400.000 membres baptisés, 1.800 pasteurs et 1.750 lieux de culte, sur une population de 10,5 millions d'habitants, soit 3,8\%.

10. L'Institut supérieur de théologie de Ouagadougou est fondé en 1996, il est une émanation (centre par extension) de la Faculté de théologie des AD de Lomé (FATAD). Il existe en Afrique, 
financés par la Mission américaine, deux autres instituts comparables à celui de Lomé, à Naïrobi et en Afrique du Sud.

11. $C f$.dans ce numéro, les précisions apportées par Sandra Fancello.

12. "Where we've been ", $A / G$ online USA, 2003 (statistiques fournies pour 2001).

13. Flamme, 25 , oct. 2000 , p. 8 . Cette importante différence pourrait résider dans la distinction opérée entre membres et adhérents. World Assemblies of God Fellowship cite le chiffre (2001) de plus de 30 millions de membres.

14. E. L. Nascimento (in "Praise the Lord and pass the cath-up ", News from Brasil, Cover story, 1995) parlait de 15 millions de membres. Ce chiffre serait passé à 17 millions aujourd'hui (cf. 3 ème congrès mondial des $\mathrm{AD}$ en août 2000 à Indianapolis, voir aussi World Assemblies of God Fellowship).

15. « Where we've been ", A/Gonline USA, 2003.

16. Voir World Assemblies of God Fellowship, mise à jour 2001.

17. Selon World Assemblies of God Fellowship, statistiques de 2001, pour l'Argentine, 610.000 et pour le Mexique, 800.000.

18. Le pasteur Pawentaoré est également membre du comité exécutif de la World Assemblies of God Fellowship (WAGF) fondée en 1989. Cette organisation regroupe toutes les AD, elle a été constituée pour répondre au besoin d'unité de l'Eglise.

19. Ce chiffre est lui fourni par : "Where we've been ", A/G online USA, 2003 (statistiques de 2001). Toujours selon ces sources, en 1990, les AD regroupaient pour l'Afrique, 2,1 millions de fidèles dans 26 pays. En 2003, les $\mathrm{AD}$ déclarent atteindre près de 10 millions de membres (cette période correspond à la décade d'évangélisation déclarée par l'ensemble des Eglises des AD). On ne peut toutefois pas attribuer, comme le fait Joël Noret, à ces campagnes d'évangélisation la seule responsabilité de la croissance du pentecôtisme. Sinon, comment rendre compte de la croissance des autres dénominations pentecôtistes en Afrique et de par le monde? Il y a bien évidemment d'autres facteurs (voir la suite du texte).

20. Flamme, 25 , oct. 2000, citant des chiffres du 3 ème congrès mondial des AD à Indianapolis.

21. Pour des développements, voir l'ouvrage p. 45 et p. 58.

22. A titre d'exemple les Assemblées de Dieu du Burkina Faso ont présidé la Commission électorale nationale indépendante (CENI) à l'occasion des élections présidentielles de décembre 1998.

23. Détails que j'ai réservés pour un travail consacré à l'analyse de la VIMAB (Vision missionnaire des Assemblées de Dieu du Burkina Faso). A ce propos, je tiens à remercier le programme IFRAIbadan qui n'a donné l'opportunité d'effectuer en avril 2003 une mission consacrée à l'action missionnaire des Assemblées de Dieu du Burkina Faso.

24. «L'Eglise des Assemblées de Dieu du Burkina Faso. Le cheminement en 75 ans », Contact, 17, p. 9 ; $75^{\text {ème }}$ anniversaire, 1996, p. 33 ; Flamme, 27, p. 12 ; voir aussi, $75^{\text {ème }}$ anniversaire, 1996, p. 34.

25. $75^{\text {ème }}$ anniversaire, 1996, p. 33.

26. «L'Eglise des Assemblées de Dieu du Burkina Faso. Le cheminement en 75 ans », Contact, 17, p. 9 ; voir aussi $75^{\text {ème }}$ anniversaire, 1996, p. 34.

27. «L'Eglise des Assemblées de Dieu du Burkina Faso. Le cheminement en 75 ans », Contact, 17, p. 9.

28. «L'Eglise des Assemblées de Dieu du Burkina Faso. Le cheminement en 75 ans », Contact, 17, p. 9 ; voir aussi : $75^{\text {ème }}$ anniversaire, 1996, p. 33.

29. $75^{\text {ème }}$ anniversaire, 1996, p. 34 ; Aperçu sur l'histoire des Assemblées de Dieu en Côte d'Ivoire, Pasteur Jean Pawentaoré Ouedraogo, p. 6 ; voir aussi, «L'Eglise des Assemblées de Dieu du Burkina Faso. Le cheminement en 75 ans », Contact, 17, p. 9 ; Flamme, 27, p. 12 ; Eglise évangélique des Assemblées de Dieu de Côte d'Ivoire, Annuaire 1995, p. 3 ; voir enfin, $75^{\text {ème }}$ anniversaire, 1996, p. 
34. Certes, pour certains pays (les "pays côtiers »), à la suite d'accords territoriaux entre les différents groupes missionnaires pentecôtistes, ces implantations furent, parfois, confinées à une partie du territoire de ces pays, il n'est reste pas moins vrai que les pasteurs mossi jouèrent un rôle notoire dans la propagation des $\mathrm{AD}$ en Afrique de l'Ouest.

30. $75^{\text {ème }}$ anniversaire, 1996, p. 33.

31. Flamme, 27, p. 12.

32. Il a travaillé à la « radio Evangile » de Lomé jusqu'en décembre 1998.

33. Rencontre en avril 2003 avec le pasteur Alpha Traoré, coordinateur de la mission française au Burkina Faso et trésorier de la VIMAB.

34. Historique des Assemblées du Dieu au Niger à l'occasion de son 10 ème anniversaire, Flamme, 25, oct-déc. 2000, p. 16-17.

35. $75^{\text {ème }}$ anniversaire, 1996, p. 33.

36. « Nouvelles de la vision missionnaire », Flamme, 9, (non daté, sans doute décembre 1996), p.

27.

37. Rencontre en avril 2003 avec le pasteur Alpha Traoré, coordinateur de la mission française au Burkina Faso et trésorier de la VIMAB.

38. Pour d'autres détails, voir Pasteur D Compaoré, « Un demi siècle au service de l'Imprimerie des Assemblées de Dieu ", Flamme, 19, p. 10 (non daté, sans doute mai 1999).

39. En bien entendu, l'histoire ne retient que les noms illustres et non pas ceux des pasteurs anonymes installés en brousse. Par ailleurs, il faut se rappeler que les relations entre les ressortissants des " pays côtiers » et les paysans migrants mossi résultent d'une longue histoire. Les Mossi sont communément considérés comme des manœuvres attachés aux plantations autochtones (pour une description de ces relations inégales $c f$. Laurent, 1995, 1998). Dans ces conditions de (parfois grandes) tensions entre les communautés, il n'est pas toujours facile, pour les responsables de pays qui accueillent ces migrants, de reconnaître à des ressortissants burkinabé leur rôle dans des institutions, fussent-elles religieuses.

40. Des «crises sorcières" sont déjà apparues à d'autres moments, celui où s'écroulent les régimes coloniaux, par exemple ( $c f$. les accusations de sorcellerie décrites dans le travaux de Marwick (1965)), ou encore, celui de l'invention de l'économie des plantations indigènes (Dozon, 1985).

41. Cette situation est peut-être moins singulière qu'il n'en paraît. A titre d'hypothèse, il pourrait s'avérer utile d'établir une base de comparaison avec l'Amérique du Sud. Ainsi par exemple l'anthropologue Nestor Garcia-Canclini montre que ce qui est propre aux sociétés latinoaméricaines est précisément l'importance et la cohabitation des réseaux d'aide et de distribution de bénéfices (établis sur des critères de filiation typiques des groupes communautaires) dans les différentes institutions et organisations de la modernité et de la démocratie (la bureaucratie de l'Etat, les entreprises, les partis politiques, les syndicats de travailleurs). Pour Garcia-Canclini la coexistence d'un système démocratique basé sur un principe électoral et un "principe de faveur » conduit à rencontrer des « éléments particulièrement anti-modernes » (Garcia-Canclini, 1995).

42. Dans cette perspective, selon André Mary, «La logique corporelle, ou ce que R. Bastide appelle 'la grammaire' des corps, emprunte donc les règles de sa syntaxe aux lois de composition de la personne. (...) Cette précision est importante car elle montre que l'opérateur effectif de la gestion des ambivalences se trouve bien ici dans la théorie plurale de la personne que l'on retrouve dans les cultures africaines, ce que d'ailleurs R. Bastide laisse en partie entendre» (Mary, $2000: 187$ ).

43. Contrairement à ce qu'affirme Joël Noret, dès la préface de l'ouvrage, la référence aux travaux de Mary Douglas est explicite. Par ailleurs, je donne une définition claire, suivie d'une description ethnographique de la conception de la personne dans la société coutumière mossi 
aux pages 260 et 261 . Cette définition est bâtie sur mes propres observations et sur celles de D. Bonnet (1988) et de E. Poulet (1969).

44. Trop précis parfois selon Frédéric Moens, mais l'épaississement empirique n'est-t-il pas le propre de notre discipline et la manière la plus sûre de rendre compte du flux des phénomènes?

45. Le reproche que m'adresse Joël Noret de ne pas discuter les catégories wébériennes est sûrement dû à son ignorance des publications que j'ai consacrées à ce propos - j'en fais pourtant état dans l'introduction du livre (Laurent, 1994). Voir surtout Laurent (1998b). Il y est très largement question " $\mathrm{du}$ groupe de la sortie du groupe», discussion de la thèse de la sécularisation que je reprends schématiquement dans le présent livre.

46. Je parlais à ce propos d'une "d'euphémisation symbolique ", pour ne pas parler d'amnésie portant sur l'origine symbolique de certaines séquences de rituels liés à l'alliance. Ainsi, par exemple, le passage par un équivalent argent permet à chaque protagoniste (responsables de cultes coutumiers, musulmans, catholiques, protestants...) du pog-puusem (salutations en vue de conclure une alliance) de réinvestir le rituel, et donc le projet d'alliance, selon son propre champ sémantique, sans donc connaître yande, la honte, par le déshonneur. A la faveur d'un surinvestissement dans le respect des formes, les parties prenantes arrivent à conclure des alliances par delà la pluralité sémantique en présence. Dès lors, la cohérence de la société mossi repose sur les effets sociaux escomptés de l'alliance et se garde de toute initiative concernant la réduction de la pluralité des univers symboliques : c'est précisément ce qu'évoque la primauté accordée à wuum taaba qui privilégie le consensus plutôt que les notions de vrai et de faux.

47. La conversion des $\mathrm{AD}$, ai-je montré, se vit d'abord comme une rupture qui n'est pas équivalente au principe de coupure utilisé par R. Bastide pour traiter au Brésil du candomblé et de umbanda. Avec ce concept, Roger Bastide veut souligner une forme de cohérence qui aide à vivre la pluralité culturelle; il y a «simultanéité de comportements différents sans conflit intérieur » (cf. Balandier, 1995b : IX.). En quelque sorte, pour les pentecôtistes mossi, le malheur rode toujours (les « forces du mal » renvoient à la fois à l'univers coutumier, aux fétiches, et à la consommation, soit aussi à la corruption) et cette perpétuelle inquiétude témoigne que ce serait précisément le «principe de coupure» (la double entente) qui constituerait une menace (officiellement du moins) dans ce cas pour un fidèle des AD.

48. Problématique que j'ai largement abordée dans mon ouvrage consacré à la ruse (Laurent, 1998b). Toutefois, comme le montre André Mary, il est à remarquer que Roger Bastide est revenu de plusieurs reprises sur le "principe de coupure », et notamment lorsqu'il traite de l'effet du processus social d'intégration des esclaves noirs (Mary, 2000 : 108).

49. Cf.la proposition de J. Noret.

50. A ce propos, voir par exemple Bajoit (2003); Dubet (1994); Dubar (2000). C'est également vers une anthropologie de "l'honneur et de la honte " qu'il faudrait également se pencher (par exemple, Pitt-Rivers, 1997, pour la version française), ainsi que vers une analyse de la notion de respect (par exemple : Ramirez, 1999 ; Zubillaga, 2003). Voir aussi Comaroff et Comaroff (2000).

51. Il existe une relation entre la logique corporelle, la théorie plurale de la personne et la gestion de l'ambivalence, où « le corps, dévoré de l'intérieur ou réceptable des dieux, fonctionne comme matrice d'identité, comme surface d'inscription et d'élucidation des composantes de l'individualité » (Mary, $2000: 186)$.

52. Dans son dernier ouvrage, André Mary (2000) discute des formes du bricolage.

53. Métaphore qui traite d'un processus identitaire ou la reconnaissance de soi implique de remettre constamment en jeux son identité.

54. Dont le nom évoque le djinn d'origine arabe.

55. Récits à la faveur desquels j'énonce les catégories du mal pour les fidèles mossi.

56. Ce qui, autrement dit, équivaut à garder actif à la fois la logique plurielle fondée sur l'inclusion des contraires et l'opposition dualiste du bien et du mal.

57. Voir livre, page 377. 


\section{AUTEUR}

\section{PIERRE-JOSEPH LAURENT}

Pierre-Joseph Laurent est anthropologue, professeur à l'Université Catholique de Louvain et membre fondateur du Laboratoire d'anthropologie prospective. Il est également associé à l'Unité de recherche «Construction identitaires et mondialisation » de l'IRD (Institut de recherche pour le développement). 